PROCEEDINGS OF THE

AMERICAN MATHEMATICAL SOCIETY

Volume 136, Number 6, June 2008, Pages 2073-2079

S 0002-9939(08)09186-7

Article electronically published on February 14, 2008

\title{
LIFTING ENDOMORPHISMS TO AUTOMORPHISMS
}

\author{
WILLIAM ARVESON AND DENNIS COURTNEY
}

(Communicated by Marius Junge)

\begin{abstract}
Normal endomorphisms of von Neumann algebras need not be extendable to automorphisms of a larger von Neumann algebra, but they always have asymptotic lifts. We describe the structure of endomorphisms and their asymptotic lifts in some detail, and apply those results to complete the identification of asymptotic lifts of unital completely positive linear maps on von Neumann algebras in terms of their minimal dilations to endomorphisms.
\end{abstract}

\section{INTRODUCTION}

We work in the category whose objects are pairs $(M, \alpha)$ consisting of a normal unit-preserving $*$-endomorphism $\alpha: M \rightarrow M$ of a von Neumann algebra $M$, and whose maps are equivariant normal $*$-homomorphisms that map unit to unit. The isomorphisms of this category are conjugacies, in which $\alpha_{1}: M_{1} \rightarrow M_{1}$ is said to be conjugate to $\alpha_{2}: M_{2} \rightarrow M_{2}$ if there is a $*$-isomorphism $\theta: M_{1} \rightarrow M_{2}$ satisfying $\theta \circ \alpha_{1}=\alpha_{2} \circ \theta$.

Consider the problem of extending an endomorphism $\alpha: M \rightarrow M$ to a *automorphism of a larger von Neumann algebra, assuming that the necessary condition $\operatorname{ker} \alpha=\{0\}$ is satisfied. In that case $\alpha$ is an isometric $*$-endomorphism of $M$, and a straightforward construction produces a unital $C^{*}$-algebra $N \supseteq M$ and a *-automorphism $\beta$ of $N$ that restricts to $\alpha$ on $M$. This extension of $\alpha$ to an automorphism of a larger $C^{*}$-algebra is unique up to natural isomorphism provided one assumes that it is minimal in the sense that $M \cup \beta^{-1}(M) \cup \beta^{-2}(M) \cup \cdots$ is norm-dense in $N$.

This procedure is effective for extending endomorphisms of $C^{*}$-algebras. But it is poorly suited to this category since there is no natural way 1 of completing the $C^{*}$-algebra $N$ to a von Neumann algebra so as to obtain a $W^{*}$-dynamical system that extends $\alpha$ except in special circumstances - the most natural circumstance being that in which $\alpha$ preserves a faithful normal state of $M$. More serious problems arise when $\operatorname{ker} \alpha \neq\{0\}$, since in that case even extensions to $C^{*}$-algebraic automorphisms cannot exist. The proper way to associate a $W^{*}$-dynamical system to an endomorphism involves the notion of lifting, a concept introduced in [2] for the broader category of unital completely positive maps, and which will be described momentarily. While liftings are typically much "smaller" than the extensions of

Received by the editors March 5, 2007.

2000 Mathematics Subject Classification. Primary 46L55, 46L40.

${ }^{1}$ It is always possible to carry out such a completion, but that construction does not give rise to a functor from injective endomorphisms to $W^{*}$-dynamical systems. 3] addresses the existence issue for $E_{0}$-semigroups acting on von Neumann algebras.

(C)2008 American Mathematical Society Reverts to public domain 28 years from publication 
isometric endomorphisms described above, they always exist within the category and they enjoy good functorial properties.

We were led to these issues by a problem involving the broader category of normal unit-preserving completely positive linear maps $\phi: M \rightarrow M$ of von Neumann algebras $M$ (UCP maps). It was shown in [2] that every UCP map $\phi: M \rightarrow M$ has an asymptotic lift, which is unique up to natural isomorphism. Naturally, one wants to identify the asymptotic lift of $\phi$ in concrete terms. In [2], the asymptotic lift of $\phi$ was identified as the tail flow of the minimal dilation of $\phi$ in "most" casesnamely those cases in which the tail flow of the dilated endomorphism has trivial kernel. But in general, the minimal dilation of $\phi$ to an endomorphism can have a nontrivial kernel, and the identification problem was left open in those cases.

The purpose of this note is to identify asymptotic lifts of UCP maps on von Neumann algebras in general. This is accomplished by first giving a description of lifts of endomorphisms, in the course of which we obtain a basic result on the structure of surjective endomorphisms of von Neumann algebras that appears to have been overlooked (Theorem 3.1). We apply these results to identify the asymptotic lift of an arbitrary UCP map in terms of its minimal dilation to an endomorphism of a larger von Neumann algebra (Theorem 4.1), thereby completing Theorem 7.1 of 2 .

In related work 4, the notion of an asymptotic lift was generalized to normal positive linear maps acting on von Neumann algebras (also see [5]). It is significant that since there is no dilation theory for positive linear maps that are not completely positive, the identification problem becomes a significant issue in such cases and has been only partially solved. Further discussion can be found in [4.

\section{LIFTING ENDOMORPHISMS}

Throughout this section, $\alpha: M \rightarrow M$ will denote an endomorphism acting on a von Neumann algebra $M$. By a $W^{*}$-dynamical system we mean a pair $(N, \beta)$, where $\beta$ is a $*$-automorphism of a von Neumann algebra $N$.

Definition 2.1. A lifting of $\alpha: M \rightarrow M$ is a triple $(N, \beta, E)$ where $(N, \beta)$ is a $W^{*}$ dynamical system and $E: N \rightarrow M$ is a unit-preserving normal $*$-homomorphism satisfying $E \circ \beta=\alpha \circ E$.

Note first that for every lifting $(N, \beta, E)$ of $\alpha$, we have

$$
E(N) \subseteq M \cap \alpha(M) \cap \alpha^{2}(M) \cap \cdots .
$$

Indeed, every element $y=E(x)$ in the range of $E$ can be written in the form $y=\alpha^{n}\left(E\left(\beta^{-n}(x)\right) \in \alpha^{n}(M)\right.$ for every $n=0,1,2, \ldots$, from which the assertion is evident.

Remark 2.2 (Nondegeneracy). A lifting $(N, \beta, E)$ of $\alpha$ is said to be nondegenerate if for every $x \in N$,

$$
E\left(\beta^{n}(x)\right)=0, \quad n \in \mathbb{Z} \quad \Longrightarrow x=0 .
$$

In general, the set

$$
K=\left\{x \in N: E\left(\beta^{n}(x)\right)=0, \quad \forall n \in \mathbb{Z}\right\}
$$

is a weak ${ }^{*}$-closed two-sided ideal in $N$ satisfying $\beta(K)=K$. Hence there is a $\beta$ fixed central projection $c \in N$ such that $K=c N$. It follows that $N$ decomposes into a sum $N=K \oplus N_{0}$, where $\left(N_{0}, \beta \uparrow_{N_{0}}, E \uparrow_{N_{0}}\right)$ is a nondegenerate lift of $\alpha$ and 
$E\left(\beta^{n}(K)\right)=\{0\}$ for every $n \in \mathbb{Z}$. In particular, every lift $(N, \beta, E)$ of $\alpha$ can be reduced to a nondegenerate lift without affecting the range of the homomorphism $E$.

Let $\alpha: M \rightarrow M$ be an endomorphism of a von Neumann algebra. The sequence of von Neumann algebras $M, \alpha(M), \alpha^{2}(M), \ldots$ decreases as $n$ increases, and their intersection

$$
M_{\infty}=\bigcap_{n=1}^{\infty} \alpha^{n}(M)
$$

is called the tail algebra of $\alpha$. The restriction of $\alpha$ to the tail algebra is a surjective endomorphism; it is an automorphism iff $\operatorname{ker} \alpha \cap M_{\infty}=\{0\}$.

Proposition 2.3. Let $\alpha: M \rightarrow M$ be an endomorphism. For every lifting $(N, \beta, E)$ of $\alpha$, the following are equivalent:

(i) For every normal linear functional $\rho \in M_{*}$, one has

$$
\lim _{n \rightarrow \infty}\left\|\rho \circ \alpha^{n}\right\|=\|\rho \circ E\| .
$$

(ii) $E(N)=M_{\infty}$.

Proof. Since $E$ is a $*$-homomorphism of von Neumann algebras, it maps the unit ball of $N$ onto the unit ball of its range. Hence (ii) is equivalent to

$$
E(\text { ball } N)=\text { ball } M_{\infty}=\bigcap_{n=1}^{\infty} \alpha^{n}(\text { ball } M) .
$$

The equivalence (i) $\Longleftrightarrow$ (ii) now follows from the more general assertion of Lemma 3.6 of 2 .

Definition 2.4. An asymptotic lift of an endomorphism $\alpha: M \rightarrow M$ is a nondegenerate lifting $(N, \beta, E)$ satisfying the conditions of Proposition 2.3 .

Remark 2.5 (Relation to asymptotic lifts of UCP maps). In [2], the term asymptotic lift refers to a related concept introduced for the broader category of UCP maps on dual operator systems. It is significant that an asymptotic lift in the sense of Definition 2.4 is also an asymptotic lift in the broader sense of Definition 3.1 of [2].

To prove that assertion, it suffices to show that if a lifting $(N, \beta, E)$ of an endomorphism $\alpha: M \rightarrow M$ satisfies the equivalent properties (i) and (ii) of Proposition 2.3. then those properties persist throughout the matrix hierarchy over $M$. Indeed, for each $n=1,2, \ldots$, the lifting $(N, \beta, E)$ of $\alpha: M \rightarrow M$ induces a natural lift $\left(M_{n} \otimes N, \operatorname{id}_{n} \otimes \beta, \operatorname{id}_{n} \otimes E\right)$ of the endomorphism $\operatorname{id}_{n} \otimes \alpha: M_{n} \otimes M \rightarrow M_{n} \otimes M$, and by examining matrix entries one finds that property (ii) persists at level $n$. Hence property (i) holds as well for every $n=1,2, \ldots$, and $(N, \beta, E)$ satisfies Definition 3.1 of 2 .

Two liftings $\left(N_{k}, \beta_{k}, E_{k}\right), k=1,2$, of an endomorphism $\alpha: M \rightarrow M$ are said to be isomorphic if there is an isomorphism of von Neumann algebras $\gamma: N_{1} \rightarrow N_{2}$ satisfying $\gamma \circ \beta_{1}=\beta_{2} \circ \gamma$ and $E_{2} \circ \gamma=E_{1}$.

Theorem 2.6. Every endomorphism $\alpha: M \rightarrow M$ of a von Neumann algebra has an asymptotic lifting that is unique up to isomorphism.

Proof. The proof of Theorem 3.2 of [2] explicitly constructs an asymptotic lift of a UCP map of that category in terms of the space of inverse sequences of that map. Since in the present context the map is an endomorphism $\alpha: M \rightarrow M$, one 
sees by inspection that the constructed asymptotic lift $(N, \beta, E)$ has the following properties: the space $N$ of inverse sequences is closed under multiplication, $\beta$ is a $*$-automorphism of that von Neumann algebra, and $E: N \rightarrow M$ is a normal *-homomorphism. Hence $(N, \beta, E)$ is an asymptotic lift in the sense of Definition 2.4. The proof of uniqueness involves similar observations.

\section{StruCture OF SURJECTIVE ENDOMORPHisms}

In this section we prove that in general, a surjective endomorphism of a von Neumann algebra admits a natural decomposition into the direct sum of a $W^{*}$ dynamical system and an endomorphism of a particularly simple kind, called a backward shift, that depends only on ker $\alpha$. That allows us to identify asymptotic lifts of endomorphisms in very concrete terms.

Let $K$ be a von Neumann algebra and consider the von Neumann algebra $\ell^{\infty}(\mathbb{N}, K)$ of all singly-infinite bounded sequences

$$
x=\left(x_{1}, x_{2}, \ldots\right), \quad x_{k} \in K .
$$

Define an endomorphism $\sigma_{+}$of $\ell^{\infty}(\mathbb{N}, K)$ as follows:

$$
\sigma_{+}\left(x_{1}, x_{2}, \ldots\right)=\left(x_{2}, x_{3}, \ldots\right), \quad\left(x_{1}, x_{2}, \ldots\right) \in \ell^{\infty}(\mathbb{N}, K) .
$$

Obviously, $\sigma_{+}$is a normal surjective unit-preserving endomorphism, and

$$
\operatorname{ker} \sigma_{+}=K \oplus 0 \oplus 0 \oplus \cdots \cong K .
$$

Such an endomorphism $\sigma_{+}$is called the backward shift based on $K$.

We can modify the backward shift $\sigma_{+}$based on $K$ in a nontrivial way by choosing an automorphism $\beta$ of another von Neumann algebra $P$ and letting $\beta \oplus \sigma_{+}$be the endomorphism of $P \oplus \ell^{\infty}(\mathbb{N}, K)$ defined by

$$
\beta \oplus \sigma_{+}: x \oplus y \in P \oplus \ell^{\infty}(\mathbb{N}, K) \mapsto \beta(x) \oplus \sigma_{+}(y) .
$$

This is a surjective endomorphism whose kernel is isomorphic to $K$, but it has a summand $P$ on which it restricts to an automorphism.

Theorem 3.1. Every normal surjective endomorphism $\alpha$ of a von Neumann algebra $M$ is conjugate to one of the form (3.2), where $\sigma_{+}$is the backward shift based on $K=\operatorname{ker} \alpha$, and where $\beta$ is the $*$-automorphism defined by restricting $\alpha$ to $P=(\mathbf{1}-c) M, c$ being the $\alpha$-fixed central projection

$$
c=\lim _{n \rightarrow \infty} c_{n},
$$

where $c_{1} \leq c_{2} \leq \cdots$ is the sequence of central projections $\operatorname{ker} \alpha^{n}=c_{n} M$.

Proof. Since ker $\alpha^{n}$ is a weak*-closed ideal in $M$ for every $n=1,2, \ldots$, it has the form $c_{n} M$ where $c_{n}$ is a central projection; and since $\operatorname{ker} \alpha^{n} \subseteq \operatorname{ker} \alpha^{n+1}$, it follows that $c_{n} \leq c_{n+1}$.

We claim: $\alpha\left(c_{n+1}\right)=c_{n}$, for every $n=1,2, \ldots$ Since $\operatorname{ker} \alpha^{k}=c_{k} M$ and $\alpha(M)=M$, this is equivalent to the assertion $\alpha\left(\operatorname{ker} \alpha^{n+1}\right)=\operatorname{ker} \alpha^{n}$. Obviously, $x \in \operatorname{ker} \alpha^{n+1} \Longrightarrow \alpha(x) \in \operatorname{ker} \alpha^{n}$. For the opposite inclusion, choose $y \in \operatorname{ker} \alpha^{n}$. Since $M=\alpha(M)$, we can find $x \in M$ such that $y=\alpha(x)$. This $x$ must satisfy $\alpha^{n+1}(x)=\alpha^{n}(\alpha(x))=\alpha^{n}(y)=0$, hence $x \in \operatorname{ker} \alpha^{n+1}$, and therefore $y=\alpha(x) \in$ $\alpha\left(\operatorname{ker} \alpha^{n+1}\right)$. These formulas $\alpha\left(c_{n+1}\right)=c_{n}$ clearly imply that the limit $c$ of (3.3) is an $\alpha$-fixed central projection. 
Let $P=(\mathbf{1}-c) M$. Note that $\alpha(P)=\alpha(\mathbf{1}-c) \alpha(M)=(\mathbf{1}-c) M=P$. Since the kernel of $\alpha$ is $c_{1} M \subseteq c M$ we have $P \cap \operatorname{ker} \alpha=\{0\}$, hence $\alpha$ restricts to a *-automorphism of $P$.

Turning now to the summand $c M$, we claim that for every $n \geq 2, \alpha^{n-1}$ restricts to an isomorphism of von Neumann algebras

$$
\alpha^{n-1}:\left(c_{n}-c_{n-1}\right) M \cong \operatorname{ker} \alpha=c_{1} M
$$

Indeed, the restriction of $\alpha^{n-1}$ to $\left(c_{n}-c_{n-1}\right) M$ is injective because $\operatorname{ker} \alpha^{n-1}=$ $c_{n-1} M$ intersects trivially with the algebra on the left. It is surjective because after iterating the formulas $\alpha\left(c_{k+1}\right)=c_{k}$ we find that

$$
\begin{aligned}
\alpha^{n-1}\left(\left(c_{n}-c_{n-1}\right) M\right) & =\alpha^{n-1}\left(c_{n} M\right)=\alpha^{n-1}\left(c_{n}\right) M=\alpha^{n-2}\left(c_{n-1}\right) M \\
& =\cdots=\alpha\left(c_{2}\right) M=c_{1} M .
\end{aligned}
$$

Now consider the von Neumann algebra $\ell^{\infty}(\mathbb{N}$, ker $\alpha)$, the algebra of all uniformly bounded sequences $y=\left(y_{1}, y_{2}, \ldots\right)$ with $y_{k} \in \operatorname{ker} \alpha$ for $k \geq 1$. Every element $x \in$ $c M$ admits a unique decomposition into a bounded sequence of mutually orthogonal central slices $x=x_{1}+x_{2}+x_{3}+\cdots$, where $x_{1}=c_{1} x$ and $x_{k}=\left(c_{k}-c_{k-1}\right) x$ for $k \geq 2$. Moreover, the preceding paragraph implies that $\alpha^{k-1}\left(x_{k}\right) \in \operatorname{ker} \alpha$ for every $k \geq 2$. Thus we can define a normal homomorphism of von Neumann algebras $\theta: c M \rightarrow \ell^{\infty}(\mathbb{N}, \operatorname{ker} \alpha)$ by

$$
\theta(x)=\left(x_{1}, \alpha\left(x_{2}\right), \alpha^{2}\left(x_{3}\right), \ldots\right), \quad x \in c M .
$$

We have also seen that for each $k \geq 2, \alpha^{k-1}$ restricts to an isomorphism from $\left(c_{k}-c_{k-1}\right) M$ to ker $\alpha$; and since $c M=c_{1} M \oplus\left(c_{2}-c_{1}\right) M \oplus\left(c_{3}-c_{2}\right) M \oplus \cdots$, it follows that $\theta$ is an isomorphism of von Neumann algebras.

One can now directly verify that $\theta \circ \alpha=\sigma_{+} \circ \theta$, where $\sigma_{+}$denotes the backward shift on $\ell^{\infty}(\mathbb{N}, \operatorname{ker} \alpha)$. We conclude that $\theta$ implements a conjugacy of the restriction of $\alpha$ to $c M$ and the backward shift based on $\operatorname{ker} \alpha$.

The $W^{*}$-dynamical system $\left(P, \alpha \uparrow_{P}\right)$ is called the automorphic summand of $\alpha$. It is clear from the preceding proof that two surjective endomorphisms are conjugate iff their automorphic summands are conjugate $W^{*}$-dynamical systems and their kernels are isomorphic von Neumann algebras.

Theorem 3.1 leads to the following description of the asymptotic lifts of arbitrary endomorpisms of von Neumann algebras.

Corollary 3.2. Let $\alpha: M \rightarrow M$ be an endomorphism with tail algebra $M_{\infty}=$ $\bigcap_{n \geq 1} \alpha^{n}(M)$. Let $K=\operatorname{ker} \alpha \cap M_{\infty}$, let $\sigma_{+}$be the backward shift acting on $\ell^{\infty}(\mathbb{N}, K)$, and let $(P, \beta)$ be the automorphic summand of $\alpha \uparrow_{M_{\infty}}$.

By Theorem 3.1, there is an isomorphism of von Neumann algebras

$$
\theta: P \oplus \ell^{\infty}(\mathbb{N}, K) \rightarrow M_{\infty}
$$

that satisfies $\theta \circ\left(\beta \oplus \sigma_{+}\right)=\alpha \circ \theta$. Let $\sigma$ be the bilateral shift acting on the von Neumann algebra $\ell^{\infty}(\mathbb{Z}, K)$ by way of $\sigma\left(x_{n}\right)=\left(x_{n+1}\right)$, and define a homomorphism $E: P \oplus \ell^{\infty}(\mathbb{Z}, K) \rightarrow M_{\infty}$ by

$$
E\left(p \oplus\left(x_{n}\right)\right)=\theta\left(p \oplus\left(x_{1}, x_{2}, \ldots\right)\right), \quad p \in P, \quad\left(x_{n}\right) \in \ell^{\infty}(\mathbb{Z}, K) .
$$

Then $\left(P \oplus \ell^{\infty}(\mathbb{Z}, K), \beta \oplus \sigma, E\right)$ is the asymptotic lift of $\alpha: M \rightarrow M$. 
Proof. It is obvious that $\left(P \oplus \ell^{\infty}(\mathbb{Z}, K), \beta \oplus \sigma\right)$ is a $W^{*}$-dynamical system and that $E$ is a homomorphism of von Neumann algebras with range

$$
E\left(P \oplus \ell^{\infty}(\mathbb{Z}, K)\right)=\theta\left(P \oplus \ell^{\infty}(\mathbb{N}, K)\right)=M_{\infty} .
$$

Moreover,

$$
\begin{aligned}
E \circ(\beta \oplus \sigma)\left(p \oplus\left(x_{n}\right)\right) & =E\left(\beta(p) \oplus\left(x_{n+1}\right)\right)=\theta\left(\beta(p) \oplus\left(x_{2}, x_{3}, \ldots\right)\right) \\
& =\theta\left(\beta(p) \oplus \sigma_{+}\left(x_{1}, x_{2}, \ldots\right)\right)=\alpha \circ \theta\left(p \oplus\left(x_{1}, x_{2}, \ldots\right)\right) \\
& =\alpha \circ E\left(p \oplus\left(x_{n}\right)\right),
\end{aligned}
$$

hence $E \circ(\beta \oplus \sigma)=\alpha \circ E$. We conclude that $\left(P \oplus \ell^{\infty}(\mathbb{Z}, K), \beta \oplus \sigma, E\right)$ is a lifting of $\alpha$ that satisfies condition (ii) of Proposition 2.3 , and it remains only to show that this lifting is nondegenerate. But if $p \in P$ and $\left(x_{n}\right) \in \ell^{\infty}(\mathbb{Z}, K)$ are such that $E\left((\beta \circ \sigma)^{k}\left(p \oplus\left(x_{n}\right)\right)=\theta\left(\beta^{k}(p) \oplus\left(x_{k+1}, x_{k+2}, \ldots\right)\right)=0\right.$ for every $k \in \mathbb{Z}$, then $\beta^{k}(p)=0$ and $x_{k+1}=0$ for every $k \in \mathbb{Z}$. The desired formula $p \oplus\left(x_{n}\right)=0$ follows.

\section{Application to UCP maps on von NeUmann ALGEBRAs}

Let $\phi: M \rightarrow M$ be a UCP map acting on a von Neumann algebra $M$. In this section we identify the asymptotic lift of $\phi$ in terms of its minimal dilation to an endomorphism of a larger von Neumann algebra. This solves the identification problem in general by strengthening Theorem 7.1 of 2 that was restricted to the case in which the minimal dilation has trivial kernel. Indeed, the following result applies to dilations of $\phi$ that are not necessarily minimal (see Chapter 8 of [1]).

Theorem 4.1. Let $\alpha: N \rightarrow N$ be an endomorphism of a von Neumann algebra and let $p \in N$ be a projection that satisfies $\alpha(p) \geq p$ and $\alpha^{n}(p) \uparrow \mathbf{1}$ as $n \uparrow \infty$. Let $M=p N p$ and let $\phi: M \rightarrow M$ be the UCP map defined by

$$
\phi(x)=p \alpha(x) p, \quad x \in M=p N p .
$$

Let $(\tilde{N}, \tilde{\alpha}, E)$ be the asymptotic lift of $\alpha$ described in Corollary 3.2. Then the asymptotic lift of $\phi$ is $(\tilde{N}, \tilde{\alpha}, \tilde{E})$, where $\tilde{E}: \tilde{N} \rightarrow M$ is the UCP map

$$
\tilde{E}(x)=p E(x) p, \quad x \in \tilde{N} .
$$

Proof. Obviously $\tilde{E}: \tilde{N} \rightarrow M$ is a UCP map and we claim $\phi \circ \tilde{E}=\tilde{E} \circ \tilde{\alpha}$. Indeed, we can use $p \alpha(p)=\alpha(p) p=p$ and $\alpha \circ E=E \circ \tilde{\alpha}$ to write

$$
\phi(\tilde{E}(x))=p \alpha(\tilde{E}(x)) p=p \alpha(p) \alpha(E(x)) \alpha(p) p=p E(\tilde{\alpha}(x)) p=\tilde{E} \circ \tilde{\alpha}(x) .
$$

Hence $(\tilde{N}, \tilde{\alpha}, \tilde{E})$ is a lifting of $\phi$. To see that it is nondegenerate, choose $x \in \tilde{N}$ such that $\tilde{E}\left(\tilde{\alpha}^{k}(x)\right)=0, k \in \mathbb{Z}$. Then for $n \geq 1$ we can apply $\alpha^{n}$ to $\tilde{E}\left(\tilde{\alpha}^{-n}(x)\right)=0$ and use $\alpha \circ E=E \circ \tilde{\alpha}$ to obtain

$$
0=\alpha^{n}\left(\tilde{E}\left(\tilde{\alpha}^{-n}(x)\right)\right)=\alpha^{n}(p) \alpha^{n}\left(E\left(\tilde{\alpha}^{-n}(x)\right)\right) \alpha^{n}(p)=\alpha^{n}(p) E(x) \alpha^{n}(p) .
$$

Since $\alpha^{n}(p) \uparrow \mathbf{1}$ as $n \uparrow \infty$, it follows that $E(x)=0$. Replacing $x$ with $\tilde{\alpha}^{k}(x), k \in \mathbb{Z}$, and using nondegeneracy of $(\tilde{N}, \tilde{\alpha}, E)$, we conclude that $x=0$.

We claim that for every $\rho \in M_{*}$,

$$
\lim _{n \rightarrow \infty}\left\|\rho \circ \phi^{n}\right\|=\|\rho \circ \tilde{E}\| .
$$


To prove (4.2), fix $\rho$ and define a normal functional $\bar{\rho} \in N_{*}$ by $\bar{\rho}(y)=\rho($ pyp). For every $x \in \tilde{N}$ we have $\rho \circ \tilde{E}(x)=\rho(p E(x) p)=\bar{\rho} \circ E(x)$, and as in the proof of formula (7.2) of [2], we obtain the following formulas for $n \geq 1$ :

$$
\left\|\rho \circ \phi^{n}\right\|=\left\|\bar{\rho} \circ \alpha^{n}\right\|, \quad\|\rho \circ \tilde{E}\|=\|\bar{\rho} \circ E\| .
$$

Since $(\tilde{N}, \tilde{\alpha}, E)$ is the asymptotic lift of $\alpha: N \rightarrow N,\left\|\bar{\rho} \circ \alpha^{n}\right\|$ converges to $\|\bar{\rho} \circ E\|$ as $n \rightarrow \infty$, and (4.2) follows. Similarly, one can promote (4.2) throughout the matrix hierarchy over $M$ exactly as in the proof of Theorem 7.1 of [2] to complete the proof of Theorem 4.1

\section{REFERENCES}

1. W. Arveson, Noncommutative dynamics and E-semigroups, Springer Monographs in Mathematics, Springer-Verlag, New York, 2003. MR1978577 (2004g:46082)

2. W. Arveson, The asymptotic lift of a completely positive map, J. Funct. Anal. (to appear), 2006. Preprint at arXiv:math.OA/0606541 v5.

3. W. Arveson and A. Kishimoto, A note on extensions of semigroups of $*$-endomorphisms, Proc. Amer. Math. Soc. 116 (1992), no. 3, 769-774. MR1098393 (93a:46133)

4. W. Arveson and E. Størmer, Asymptotic lifts of positive linear maps, 2006. Preprint at arXiv:math.OA/0611401.

5. E. Størmer. Multiplicative properties of positive maps, Math. Scand. 100 (2007), 184-192. MR2331197

Department of Mathematics, University of California, Berkeley, California 94720

E-mail address: arveson@math.berkeley.edu

Department of Mathematics, University of California, Berkeley, California 94720

E-mail address: djc@math.berkeley.edu 